\title{
Human umbilical cord mesenchymal stem cells improve the immune-associated inflammatory and prothrombotic state in collagen type-II-induced arthritic rats
}

\author{
JIAN GU ${ }^{1}$, WEI GU ${ }^{1}$, CHUANMING LIN $^{1}$, HAO GU $^{2}$, WEI WU ${ }^{1}$, JIE YIN ${ }^{1}$, JUN NI $^{1}$, XIAOPING PEI ${ }^{1}$,

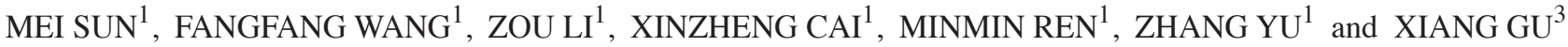 \\ ${ }^{1}$ Department of Hematology, Northern Jiangsu People's Hospital, Yangzhou, Jiangsu 225001; \\ ${ }^{2}$ Jiangsu Province Brain Hospital, Nanjing, Jiangsu 210000; ${ }^{3}$ Department of Cardiology, \\ Northern Jiangsu People's Hospital, Yangzhou, Jiangsu 225001, P.R. China
}

Received November 18, 2014; Accepted August 20, 2015

DOI: $10.3892 / \mathrm{mmr} .2015 .4394$

\begin{abstract}
Human umbilical cord mesenchymal stem cells (hUC-MSCs) hold great potential in the search for therapies to treat refractory diseases, including rheumatoid arthritis (RA), due to their potential regenerative ability and extensive source. However, the role of hUC-MSCs in vivo and the repair mechanisms for RA remain to be fully elucidated. The present study aimed to determine whether hUC-MSCs exert immunomodulatory effects and have anti-inflammatory capabilities in the treatment of embolisms. Following the transplantation of hUC-MSCs into collagen type II-induced arthritic (CIA) model rats, magnetic resonance imaging (MRI) in vivo was performed, and the levels of interleukin (IL)-1, IL-17, tumor necrosis factor (TNF)- $\alpha$, vascular endothelial growth factor (VEGF), tissue factor (TF), CD $4^{+} \mathrm{CD} 25^{+} \mathrm{T}$ cells (Treg) and antithrombin (AT) were measured. Bromodeoxyuridine staining was performed for histopathological examinations. As revealed by immunofluorescence and MRI experiments, the injected hUC-MSCs preferentially migrated to the inflammatory joint sites of the rats. The Treg cell percentage and AT levels in the hUC-MSC-treated group were markedly increased, whereas the levels of IL-1, IL-17, TNF- $\alpha$, VEGF and $\mathrm{TF}$ were decreased compared with those in the CIA model group. The values determined for these parameters in the hUC-MSC-treated group returned to approximately the identical values as those of the control group on day 35 post-therapy. Superparamagnetic iron oxide nanoparticles (SPIONs) may serve as an effective, non-invasive method for tracking
\end{abstract}

Correspondence to: $\mathrm{Dr}$ Jian Gu, Department of Hematology, Northern Jiangsu People's Hospital, 98 Nantong West Road, Yangzhou, Jiangsu 225001, P.R. China

E-mail: maolujiu918@163.com

Key words: rheumatoid arthritis, human umbilical cord mesenchymal stem cells, magnetic resonance imaging, superparamagnetic iron oxide nanoparticles, inflammatory thrombosis transplanted cells in vivo. The present study provided direct evidence that hUC-MSCs in the CIA rat model migrated to the inflammatory joint sites, effectively promoting recovery from collagen type II damage and thereby improving the immune-associated prothrombotic state.

\section{Introduction}

Rheumatoid arthritis (RA) is a chronic autoimmune and highly destructive disease of the joints, characterized by persistent synovitis, cartilage degradation and bone erosion. At present, RA remains incurable and patients with RA are often refractory to a number of drugs (1). Although the exact pathogenesis of RA remains to be fully elucidated, numerous previous studies have demonstrated that abnormalities in $\mathrm{T}$ and $\mathrm{B}$ lymphocytes, and macrophages are pivotal in its pathogenesis. Genetic and environmental factors exert orchestrated roles. For example, B cells aggravate the pathogenic process through autoantibody and cytokine production. Antigen-activated $\mathrm{CD}^{+} \mathrm{T}$ cells amplify the immune response. Synovitis is associated with the local activation of mononuclear cells, formation of new blood vessels and the release of cytokines, particularly tumor necrosis factor (TNF)- $\alpha$, interleukin (IL)-6, IL-1 and IL-17 $(2,3)$. In parallel, inflammatory responses increase the levels of procoagulant factors, and the natural anticoagulant pathways and fibrinolytic activities are inhibited, leading to a thrombotic tendency. Previous studies identified a previously unrecognized role for platelets and their activation-induced microparticles in autoimmune inflammatory arthritis. It appears that there may be very complex interactions occurring between inflammation and hemostasis. Coagulation augments inflammation, leading to a positive feedback mechanism. Inflammation-induced thrombosis responded to immunosuppressants, statins, anticoagulants and antiplatelet agents, however, a decreased effectiveness of the drugs over time and adverse reactions were often observed $(4,5)$. The initial consensus guidelines for stem cell transplantation in autoimmune diseases were published in 1997, and the immunomodulatory potential of stem cells was subsequently confirmed (6-8). To the best of our knowledge, only a few 
previous studies have investigated the role of human umbilical cord mesenchymal stem cells (hUC-MSCs) in inflammatory thrombosis in RA (9). In addition, an important goal in this research is to develop an in vivo, non-invasive tracking method for determining the retention, migration and effects of implanted hUC-MSCs. Magnetic nanoparticles, particularly superparamagnetic iron oxide nanoparticles (SPIONs), have long been investigated as a contrast agent for magnetic resonance imaging (MRI) (10). In the present study, the rat model of collagen type II-induced arthritis (CIA) was established in order to assess the efficacy of hUC-MSCs in regulating the immune response and in decreasing inflammatory thrombosis, and to further investigate the mechanism of repair mediated by hUC-MSCs in CIA through tracking the cells in vivo and in vitro.

\section{Materials and methods}

General experimental process. A total of ninety female Sprague Dawley rats (age, five weeks; average weight, $175 \pm 10 \mathrm{~g}$ ) were purchased from the Nanjing University Model Animal Research Center (Nanjing, China). The rats were housed in clean cages under hygienic conditions and were allowed to acclimatize for at least 7 days prior to the experiment. The skin of each rat was shaved and cleaned with iodine solution (Nanjing Zhonghe Pharmaceuticals, Nanjing, China) prior to the procedure. A general anesthesia was induced by means of intramuscular injection of ketamine (30 mg/kg; Heng Rui Pharmaceuticals, Lianyungang, China) to minimize the suffering of the rats during the experiments. Following each procedure, the rats were clinically observed in their cages with respect to food, water, body weight, temperature and their general behavior. The protocols were approved by the ethics committee of Northern Jiangsu People's Hospital (Jiangsu, China). All studies were performed at the Yangzhou Institute of Hematology (Yangzhou, China) and at the Animal Experiment Center of Yangzhou and Southeastern University.

Reagents. Chicken collagen type II (CII), complete Freund's adjuvant, bromodeoxyuridine (BrdU), mouse anti-rat BrdU monoclonal antibody (cat. no. B253; 1:500 dilution), goat anti-mouse fluorescein isothiocyanate (FITC) polyclonal antibody (cat. no. F5387; 1:64 dilution) and horseradish peroxidase-labeled goat anti-mouse IgG polyclonal antibody (cat. no. A9917; dilution, 1:150) were purchased from Sigma-Aldrich (St. Louis, MO, USA). ELISA kits for the detection of IL-1 (cat. no. 24494), IL-17 (cat. no. 301291), TNF- $\alpha$ (cat. no. 24835), vascular endothelial growth factor (VEGF; cat. no. 83785), tissue factor (TF; cat. no. 14066), antithrombin (AT; cat. no. DSPC10) were purchased from R\&D Systems (Minneapolis, MN, USA). FITC mouse anti-rat CD4 monoclonal antibody (cat. no. 554837; 1:200 dilution), FITC mouse anti-rat CD11b monoclonal antibody (cat. no. 554982; 1:200 dilution) and phycoerythrin (PE)-mouse anti-rat CD25 monoclonal antibody (cat. no. 554866; 1:200 dilution) were purchased from BD Biosciences (San Jose, CA, USA). SPIONs were prepared at the Chemistry and Chemical Engineering College of Yangzhou University (Yangzhou, China).
CIA model. The experiments were performed in 5-week-old female Sprague-Dawley rats (weighing, 175 10 g; Nanjing Medical University, Nanjing, China). A total of 120 rats were randomly separated into four groups: Control group (C), the CIA model group (M), the SPION and BrdU-unlabeled UC-MSCs group (U) and the SPION and BrdU-labeled UC-MSCs group (L). The CIA model was induced, as described previously (11). With the exception of the $\mathrm{C}$ group, each rat was intradermally injected with $0.1 \mathrm{ml}(0.25 \mathrm{mg}) \mathrm{CII}$ into its right hind-paw on day 1 , and an identical dose of CII was injected into the rat tail and left hind plantar on day 21 to reinforce the extent of the immune damage.

hUC-MSCs labeling. The solution, containing SPIONs, was added to $2 \times 10^{6} / \mathrm{ml}$ hUC-MSCs (obtained from the Human Umbilical Cord Mesenchymal Stem Cell Bank of Taizhou, Taizhou, China) and co-cultured for $12 \mathrm{~h}$. The final concentration of SPIONs was $25 \mu \mathrm{g} \mathrm{Fe} / \mathrm{ml}$. An identical dose of hUC-MSCs was added to $15 \mu \mathrm{M}$ BrdU and co-cultured for $48 \mathrm{~h}$. All experiments were performed at least in triplicate.

Prussian blue staining. The UC-MSCs were washed three times with phosphate-buffered saline (PBS) and fixed for $30 \mathrm{~min}$ with glutaraldehyde (Sinopharm Chemical Regent, Shanghai, China) at room temperature. The cells were washed three times with $\mathrm{PBS}$, prior to incubation with $20 \%$ potassium ferrocyanide (Sinopharm Chemical Regent) and 20\% hydrochloric acid for $20 \mathrm{~min}$ at room temperature. They were subsequently washed once in deionized water and were dehydrated through a graded alcohol series (70, 80, 90 and 95\%). Prussian blue stain (Sinopharm Chemical Regent) was applied to determine the presence of iron particles in the SPION-labeled hUC-MSCs under a light microscope (CH30RF200; Olympus, Tokyo, Japan). The cells positively stained with the Prussian blue stain were quantified.

Immunocytochemical staining. The hUC-MSCs were transferred to cling film (Sigma-Alrich), washed three times with PBS and incubated in $3 \% \mathrm{H}_{2} \mathrm{O}_{2}$ for $48 \mathrm{~h}$. The cells were subsequently placed into a $20 \% \mathrm{HCl}$ solution at room temperature. Mouse anti-BrdU monoclonal antibody (dilution, 1:500) and subsequently horseradish peroxidase-labeled goat anti-mouse IgG (dilution, 1:150) were in turn added to the film. Following washing, the cells with PBS, positively stained with BrdU stain were counted under a fluorescence microscope (DX-UCB; Olympus).

Cell transplantation. Aliquots of $1 \times 10^{6}$ hUC-MSCs ( $0.5 \mathrm{ml}$ cell suspension) were injected into the tail vein in the $\mathrm{U}$ and L groups one week following the second CII injection. Alternatively, an equal volume of saline was injected into the tail vein of the $\mathrm{M}$ and $\mathrm{C}$ groups.

MRI examination in vivo. The 7.0 T Micro-MRI system (Bruker Pharma, Siemens Medical Systems, Erlangen, Germany) was used to detect SPION-positive cells on days 7, 21 and 35 following the injection of labeled hUC-MSCs. The hind limbs of the rats in group $\mathrm{L}$ were positioned in complete extension and located in the center of a standard wrist coil so that the long axis of the joint was parallel with the 
MRI unit table. MRI was subsequently performed in the transverse plane of the joints to ensure anatomic reproducibility of the image position, and to allow correlations to be made between the histological slices. The following parameters were used: Three inches of the coil, T2 $\mathrm{x}$ weighted imaging (WI), a thickness of $1 \mathrm{~mm}$ with no interval gap, a repetition time of $200.0 \mathrm{msec}$, an echo time of $3.0 \mathrm{msec}$, a fast low-angle shot, and anisotropy values as follows: Fractional anisotropy, 30.0 deg.; matrix, 256 x 256; and field of view, $70.0 \mathrm{~mm}$.

ELISA analysis. Under anesthesia with 3\% sodium pentobarbital (Sigma-Aldrich), the abdominal veins of the rats were perforated and $2 \mathrm{ml}$ venous blood was obtained. Blood was collected on days 7,21 and 35 following treatment and centrifuged at $1,400 \mathrm{x} \mathrm{g}$ at $4^{\circ} \mathrm{C}$ for $10 \mathrm{~min}$. The serum was further extracted in order to measure the levels of IL-1, IL-17, TNF- $\alpha$, VEGF, TF and AT using an ELISA kit, according to the manufacturer's instructions (R\&D Systems).

Flow cytometry. On days 7, 21 and 35 following treatment, whole venous blood of the rats was collected in tubes containing $3.8 \%$ sodium citrate. Blood cells were incubated with FITC-CD4 monoclonal antibody, FITC-CD11b monoclonal antibody or PE-CD25 monoclonal antibody in different tubes for $15 \mathrm{~min}$ in the dark. Hemolysin (BD Biosciences) was added to each tube to eliminate the red blood cells. Subsequently, the expression of T-regulatory (Treg) cells was measured by flow cytometric analysis (FCM). FCM experiments were performed on $1 \times 10^{5}$ cells and repeated at least three times.

Indirect immunofluorescence. On days 7, 21 and 35 following treatment, batches of rats were anesthetized with $3 \%$ sodium pentobarbital and following collection of venous blood, knee joints, heart, liver, spleen, lung and kidney were excised and fixed in $10 \%$ formaldehyde solution prior to paraffin embedding. The tissue sections were incubated with anti-BrdU monoclonal antibody (dilution, 1:500), and subsequently with goat anti-mouse IgG-FITC (dilution, 1:64) at room temperature. The cells positively stained with the BrdU were washed with PBS, and their distribution in tissues was subsequently assessed by counting under a fluorescence microscope (DX-UCB; Olympus).

Statistical analysis. The data from all quantitative assays are expressed as the mean \pm standard deviation. Statistical analysis were performed using SPSS 17.0 (SPSS, Inc., Chicago, IL, USA). Comparisons between groups were performed using one-way analysis of variance. $\mathrm{P}<0.05$ was considered to indicate a statistically significant difference.

\section{Results}

General state of the rats and the gross appearance of the hind legs. All rat models of CIA exhibited a markedly decreased appetite, reduced activity, listlessness, a slow weight gain and dull hair. On day 2 following the initial CII injection, skin petechiae appeared and mild swelling occurred in the homolateral joint (in the paw, ankle and knees). From day 7 following the CII injection, the swelling appeared to become progressively more aggravated, subsequently spreading to the contralateral limbs. The symptoms exhibited were typically the most severe on day 21 , following the second CII injection (compare the normal appearance of the rat hind paw in the $\mathrm{C}$ group; Fig. $1 \mathrm{~A}-\mathrm{C}$ ). Following treatment of the UC-MSCs with or without SPION labeling, the rats exhibited less synovial thickening, an increased incidence of cartilage defects and tissue edema compared with those in group M (Fig. 1A-C).

In vivo MRI tracking of SPION-labeled hUC-MSCs. SPION-labeled hUC-MSCs, which are illustrated as the attenuated (dark) signal (Fig. 1D-F), were initially detected in the rat joints by in vivo MRI on day 2 following the implantation of the hUC-MSCs. On day 7, the most marked signal attenuation in the knee was observed in the SPION-labeled group (Fig. 1D), and subsequently the attenuated signal was gradually reduced up to 35 days (Fig. 1E and F). However, a similar signal and pathological changes failed to be identified in the heart, liver, spleen, lung, kidney and other organs of the identical group.

Evaluation of Prussian blue staining of hUC-MSCs in vitro. Blue iron particles were identified in the cytoplasm of almost every labeled UC-MSC. Certain labeled hUC-MSCs were congregated into groups (Fig. 2A-1). Prussian blue staining was positively taken up by $>98 \%$ of the cells.

BrdU labeling of the hUC-MSCs in vitro. Tan-colored particles were identified in the labeled hUC-MSC nuclei (Fig. 2B-1, 2). The uptake of the BrdU stain was $>97.5 \%$.

Cell viability of hUC-MSCs as determined by a trypan blue exclusion assay. The cell viability, as analyzed using the Trypan blue exclusion assay, was determined to be 99 and $98 \%$ in groups $\mathrm{U}$ and $\mathrm{L}$, respectively. No statistical differences in cell viability were identified between the hUC-MSC-treated groups.

Indirect immunofluorescence analysis of the tissue samples. The most significant fluorescence intensity of the inflammatory joints in the BrdU-labeled group was observed on day 7 following the injection of the hUC-MSCs. Subsequently, the fluorescence intensity was gradually reduced over time. The mean fluorescence intensity was similar in the two hUC-MSC-treated groups. With the exception of the inflammatory joints, immunofluorescence was not detected in other organs, including the liver, kidney, lung, spleen and heart, which suggested that hUC-MSCs failed to accumulate in these organs (Fig. 3).

Expression of the Treg cells in the different groups. The percentage of Treg cells identified in the lymphocytes of the peripheral blood in group $\mathrm{M}$ was significantly lower compared with that in the $\mathrm{C}$ group ( $\mathrm{P}<0.05$; Fig. $4 \mathrm{~A})$. In groups $\mathrm{U}$ and $\mathrm{L}$, the percentage of Treg cells was higher compared with that in the $\mathrm{C}$ group at 1 and 3 weeks following hUC-MSC transfusion. However, at 5 weeks post-treatment, no differences in the percentages of the Treg cells were identified. Furthermore, the percentage of Treg cells in the CIA model group $\mathrm{M}$ was lower compared with that in group $\mathrm{C}$ and in the 

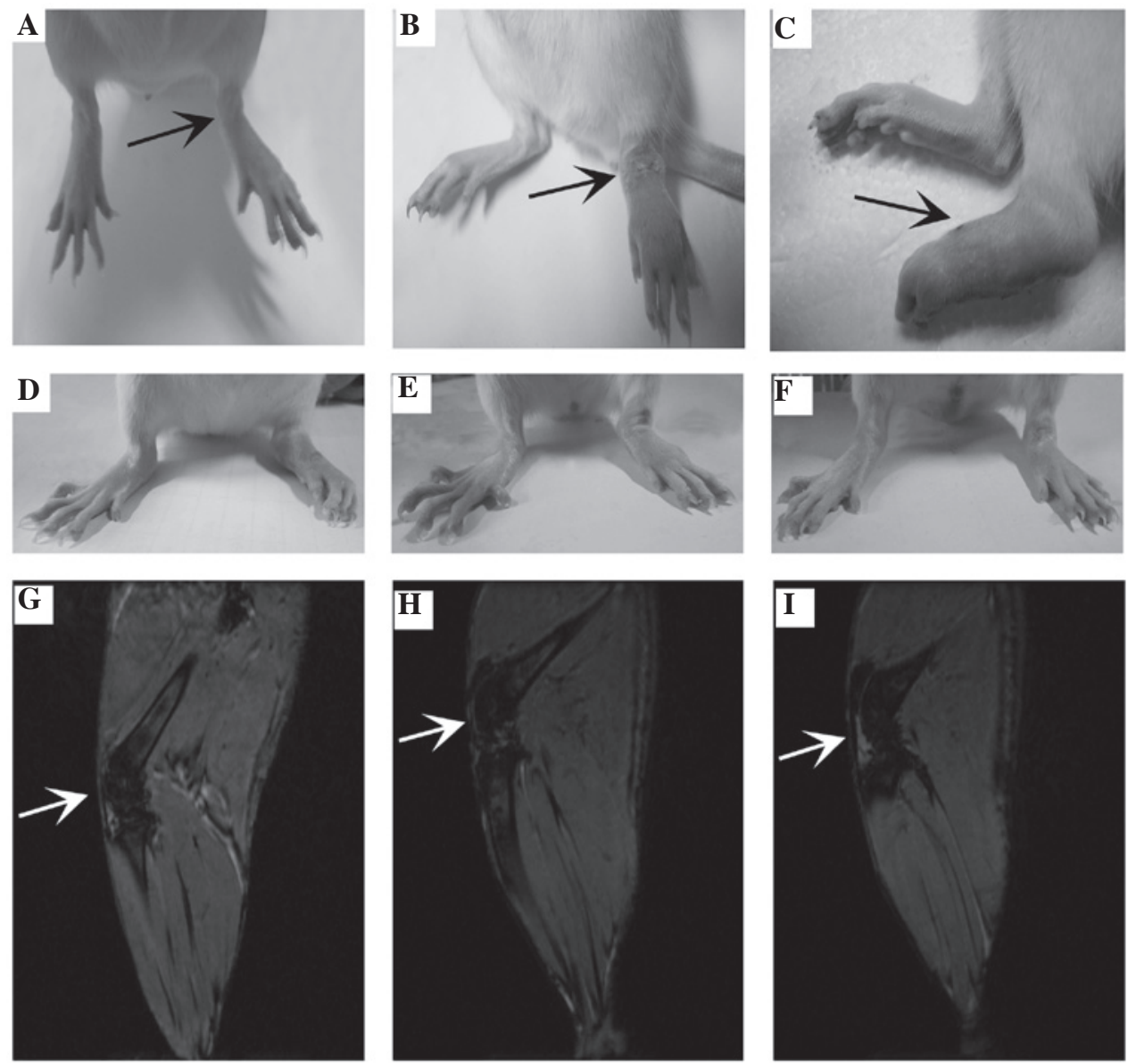

Figure 1. Changes in the gross appearance and magnetic resonance images of the rats in the superparamagnetic iron oxide nanoparticle-labeled hUC-MSC group. (A) The normal appearance of the rat hind paw in the control group C. (B and C) The skin petechiae and joint swelling in the collagen type II-induced arthritic model group M are shown. (D-F) Following treatment of the rat hind legs with hUC-MSCs, fewer skin petechiae, joint swelling and tissue edema were observed compared with those of group M during the follow-up. (G) The most marked signal attenuation of the knee was observed in group L on day 7 following treatment. (H and I) The signal attenuation was gradually reduced up to 35 days. hUC-MSCs, human umbilical cord mesenchymal stem cells.

two hUC-MSC-treated groups, $\mathrm{U}$ and $\mathrm{L}$, throughout the entire course of the experiment (Fig. 4B-F).

Levels of cytokines in the different groups. The levels of IL-1, IL-17, TNF- $\alpha$ and VEGF in group M were significantly higher compared with those of the hUC-MSC-treated groups U and L, and group $\mathrm{C}$, at 1,3 and 5 weeks following treatment $(\mathrm{P}<0.05)$. The experimental parameters for group $\mathrm{C}$ were lowest during week 1 and were similar to those of the hUC-MSC-treated group at 5 weeks post-treatment $(\mathrm{P}<0.05$; Fig. 5). The level of $\mathrm{TF}$ in group $\mathrm{M}$ was significantly higher compared with that in the hUC-MSC-treated groups at 1, 3 and 5 weeks post-treatment. By contrast, the level of AT in group M was significantly lower compared with that of the control group. The levels of AT in the hUC-MSC-treated groups increased gradually following the treatment throughout the duration of the experiment, and rose up to a level similar to that in the control group at 5 weeks post-treatment (Fig. 6).

\section{Discussion}

The present study provided direct evidence that hUC-MSCs injected into rat models of CIA migrated to the inflammatory joints, and also effectively promoted the recovery from CII damage, improving the immune inflammatory prothrombotic status.

The CIA model, similar to RA, exhibits a chronic and progressive disease course. Although there are different types of models for RA, the CIA model is the most practical and has been used extensively in research focusing on immunospecific RA. RA and CIA are associated with the expression of certain major histocompatibility class II genes. Furthermore, the CIA model provides a convenient means to investigate autoantibodies, including antibodies which are specific for the CII antigen and rheumatoid factors in the blood. Immunohistopathological investigations of the affected joints revealed a predominance of macrophages/fibroblasts, with a marked infiltration of activated $\mathrm{T}$ cells and granulocytes $(1,12)$. In the present study, rat models of CIA exhibited different degrees of hind limb congestion, edema, movement disorder, and no obvious self-limiting remission during the 5 weeks following the establishment of the model. An MRI revealed a normal signal intensity and significantly thickened synovia in the CIA group. The levels of IL-1, IL-17, TNF- $\alpha$ and VEGF in the CIA group were markedly higher compared with those in the control group at 1,3 and 5 weeks post-treatment. These results supported that the CIA model effectively mimicked an RA manifestation, and therefore is a useful tool for investigating potential therapeutic strategies 

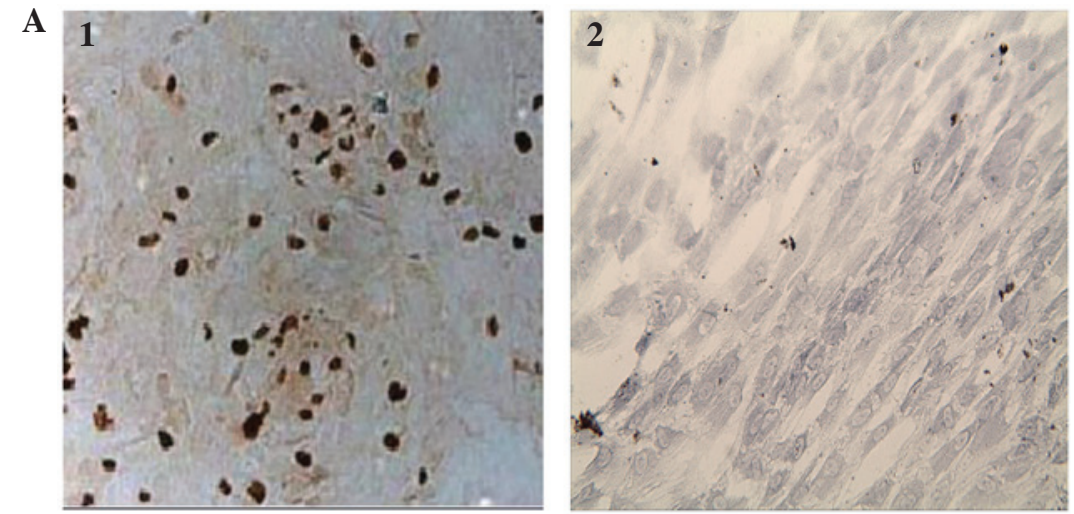

B
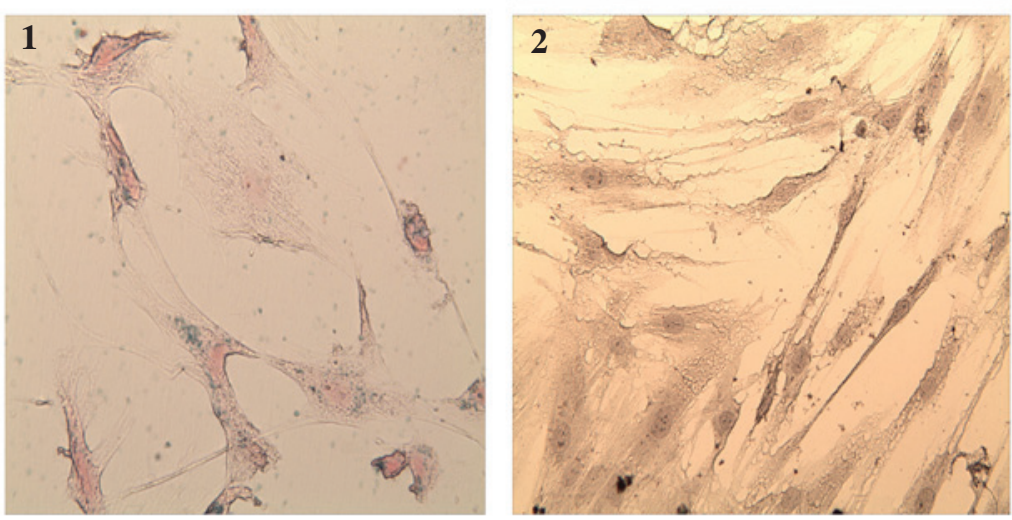

Figure 2. In vitro labeling of hUC-MSCs (magnification, x200). (A-1) hUC-MSCs with Prussian blue staining exhibited a greater number of blue particles in the cytoplasm of group L. (A-2) The identically colored particles were not observed in the unlabeled group U. (B-1) UC-MSCs with BrdU staining exhibited a greater number of tan-colored particles in the nuclei of group L. (B-2) The tan-colored particles were not observed in the unlabeled group. hUC-MSCs, human umbilical cord mesenchymal stem cells; group U, SPION- and BrdU-unlabeled UC-MSCs; group L, SPION- and BrdU-labeled UC-MSCs; SPION, superparamagnetic iron oxide nanoparticle; BrdU, bromodeoxyuridine.

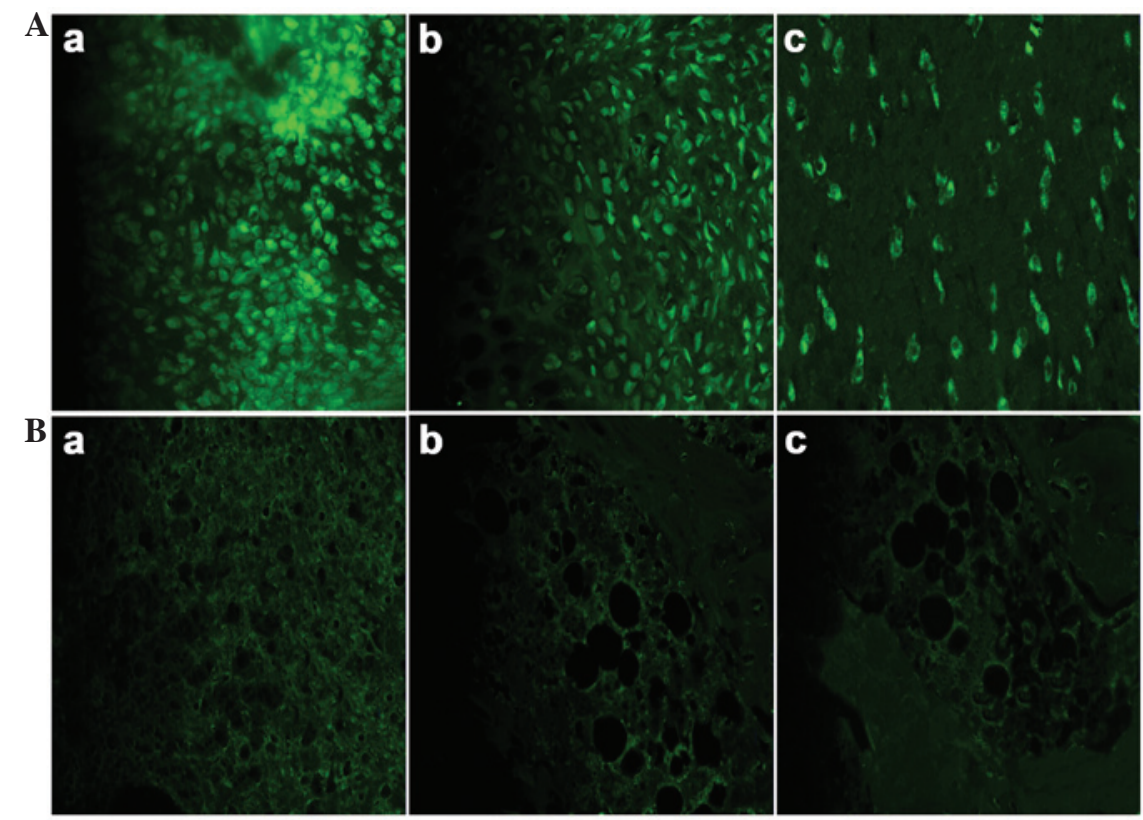

Figure 3. Indirect immunofluorescence images of the joint tissue (magnification, $\mathrm{x} 40$ ). (A, a) The strongest fluorescence intensity and the highest fluorescence density in the inflammatory knee was observed on day 7 following injection of the BrdU-tagged hUC-SCs. (A, b and c) The fluorescence intensity in the inflammatory joints was diminished over time. $(\mathrm{B}, \mathrm{a}-\mathrm{c})$ Indirect immunofluorescence in the inflammatory knee was not observed in the BrdU-unlabeled group over the identical time period. BrdU, bromodeoxyuridine.

against RA. The inflammatory activity status and joint function of the CIA model rats appeared to improve 1 week following
hUC-MSC treatment, which indicated that hUC-MSCs may be potentially useful for treating RA. 
A

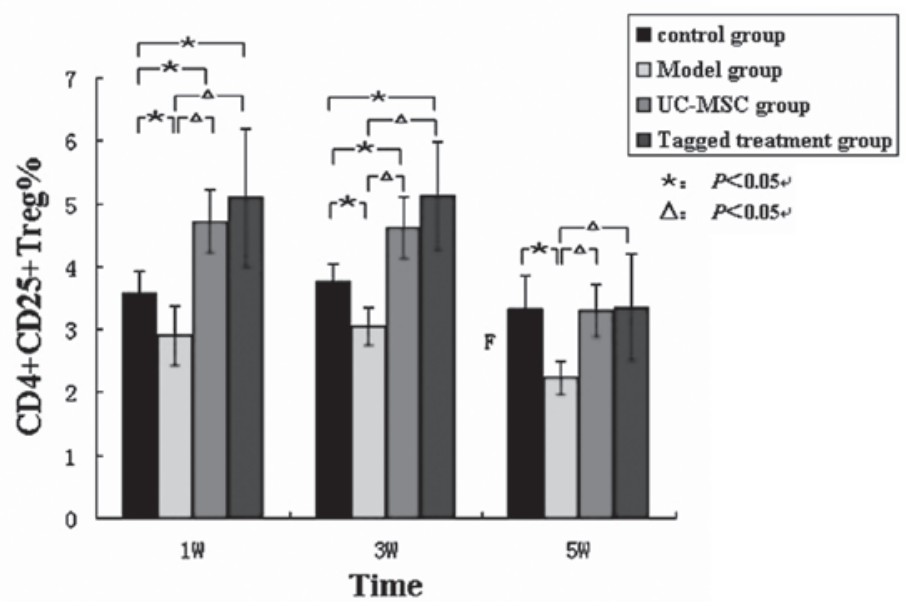

B

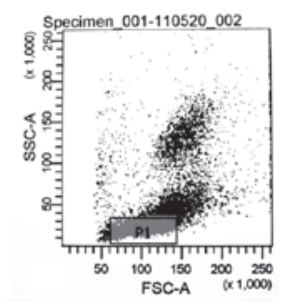

C

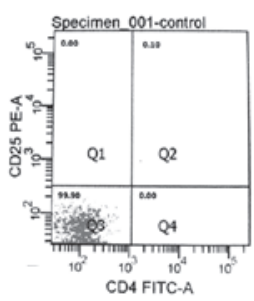

D

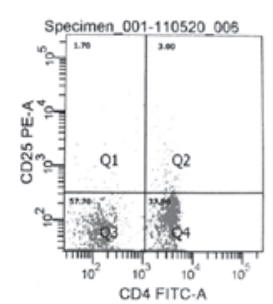

$\mathbf{E}$

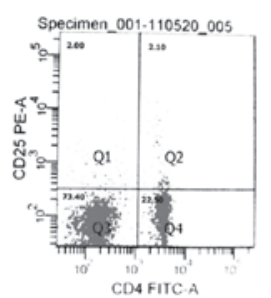

F

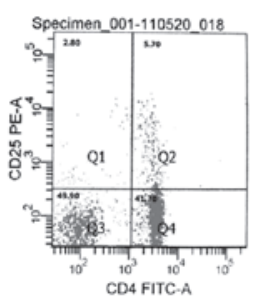

Figure 4. Expression of the Treg cells between all groups. (A) Flow cytometric analysis demonstrated that the percentage of Treg cells in the CIA model group $\mathrm{M}$ was significantly lower compared with that in the control group $\mathrm{C}$ and the two hUC-MSC-treated groups, U and L, at the different time points. ${ }^{\Delta} \mathrm{P}<0.05$, compared with the hUC-MSC-treated groups; ${ }^{*} \mathrm{P}<0.05$, compared with the blank control group. Representative flow cytometric analysis data at the identical time points were assessed. (B) The lymphocytes of the frame; the percentages of Treg cells were measured as (C) $0.1 \%$ (the negative control group); (D) $3.00 \%$ (the blank control group C); (E) 2.10\% (group M); and (F) 5.7\% (group U). Treg cells, CD4 ${ }^{+}$CD25 ${ }^{+}$T cells; W, week, hUC-MSC, human umbilical cord mesenchymal stem cell; FSC, forward scatter, SSC, side scatter; FITC, fluorescein isothiocyanate; PE, phycoerythrin; CIA, collagen type II-induced arthritic; W, weeks.

A lack of understanding of how stem cells exert their therapeutic effects is a major obstacle in regenerative medicine. At present, nanoparticles are used for various biomedical applications in which they facilitate laboratory diagnostics and therapeutics (13). By contrast with conventional MRI, which is performed using extracellular contrast agents, SPION-enhanced macrophage MRI, which is based on the non-invasive, in vivo assessment of macrophage infiltration in infected synovia, may accurately monitor the progression of joint inflammation during treatment (14). SPIONs elicit marked T2 x WI measurements in the scanned images, and T2 $x$ WI relaxation effects and a homogenous magnetic field lead to signal attenuation. The extent of the reduction in the signal is usually associated with the quantity of the labeled stem cells (15). In the present study, SPIONs combined with 7.0T MRI were successfully used for tracking the labeled hUC-MSCs. Furthermore, previous studies revealed that the optimal concentration of the SPION-labeled stem cells was $\sim 25 \mu \mathrm{g} / \mathrm{ml}$, since a low concentration of Feridex was insufficient for allowing efficient labeling, whereas a high concentration (44.8 $\mu \mathrm{g} \mathrm{Fe} / \mathrm{ml}$ ) was toxic to the cells (16). Based on these results, a concentration of Feridex $(25 \mu \mathrm{g} / \mathrm{ml})$ was used in the present study, which was low in toxicity, while allowing for efficient labeling. The trypan blue staining revealed that the viability of the UC-MSCs in the SPION-labeled group was $>90 \%$, which was sufficient for the treatment. Therefore, the use of MRI in vivo to track the hUC-MSCs with SPION proved to be successful, in view of the high sensitivity and specificity of the method in the assessment of joint disorders.

Immunofluorescence analysis revealed that the results of the BrdU-tagging experiment in vitro were consistent with those of the in vivo MRI tracking analysis of the hUC-MSCs. The fluorescence intensity of the inflammatory hind limbs reached a maximum on day 7 following the injection of the cells, which demonstrated that a large number of the UC-MSCs had migrated to the hind limbs. Furthermore, the fluorescence intensity was decreased over time, indicating that the accumulation of the hUC-MSCs into the inflammatory joints was reduced. By contrast, no immunofluorescence was detected in the liver, kidney, lung, spleen or heart, demonstrating that these tissues failed to exhibit any retention of the hUC-MSCs. A previous study confirmed that Treg cells exert an important role in peripheral immune tolerance and in the prevention of pathogenic autoimmunity in RA (17). In the present study, the quantity of the Treg cells in the CIA model group was lower compared with that of the hUC-MSC-treated groups over the period of 5 weeks. These results suggested that treatment with hUC-MSCs may lead to an increase in the number of Treg cells.

Previous studies suggested that IL-1 exerts an important role in the processes of bone erosion and cartilage destruction associated with RA, by regulating the expression of other inflammatory mediators. IL-1-induced destruction of the extracellular matrix accelerated RA joint destruction and promoted coagulation by downregulating the expression of thrombomodulin 

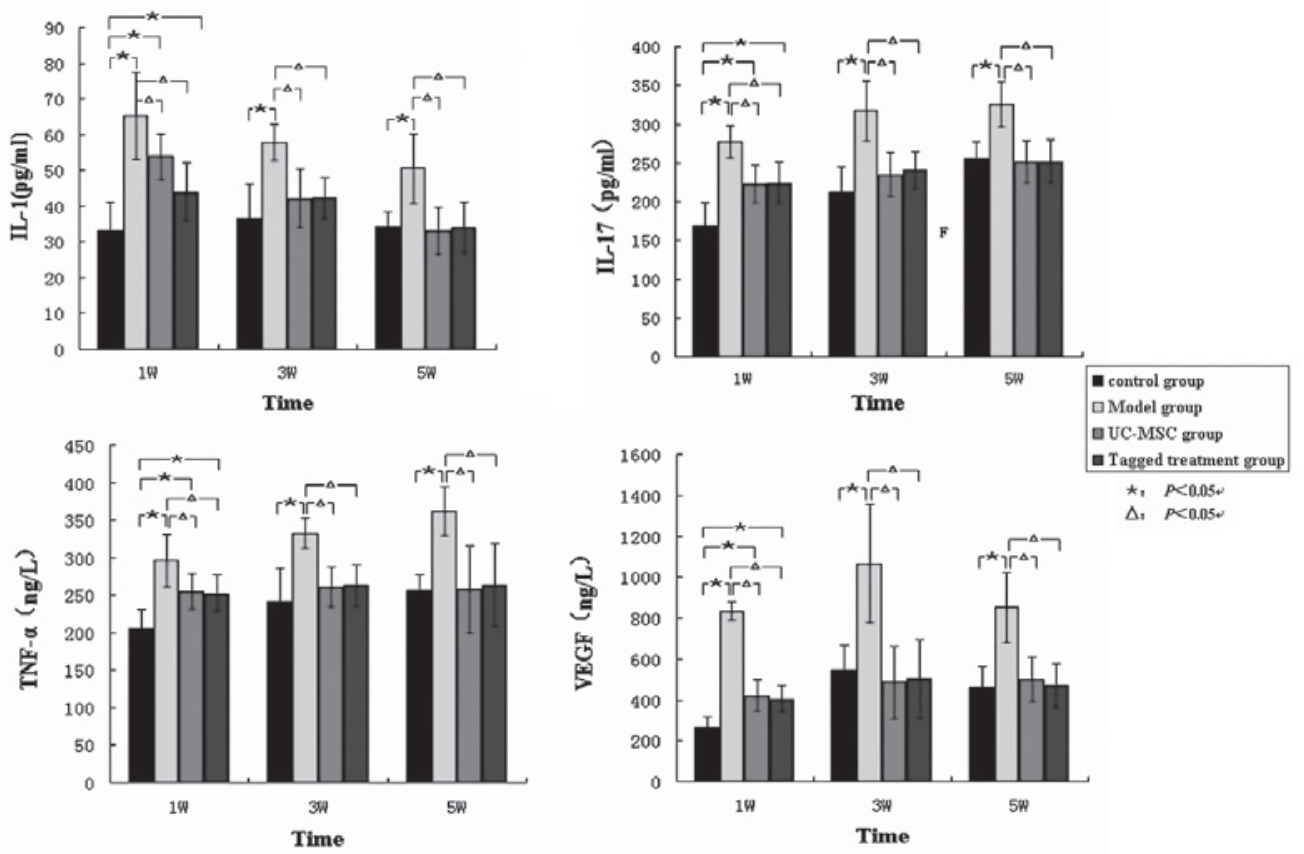

Figure 5. Levels of the serum inflammatory cytokines in the rats throughout the entire duration of the experiment. The levels of IL-1, IL-17, TNF- $\alpha$ and VEGF in group $\mathrm{M}$ were significantly higher compared with those in the UC-MSC-treated groups U and L, and the control group C. However, the levels of these inflammatory cytokines were similar between the hUC-MSC-treated groups and the control group. ${ }^{*} \mathrm{P}<0.05$, compared with the blank control group; ${ }^{\Delta} \mathrm{P}<0.05$, compared with the hUC-MSC-treated groups. IL, interleukin; TNF- $\alpha$, tumor necrosis factor- $\alpha$; VEGF. endothelial cell growth factor; hUC-MSCs, human umbilical cord mesenchymal stem cells; W, weeks.
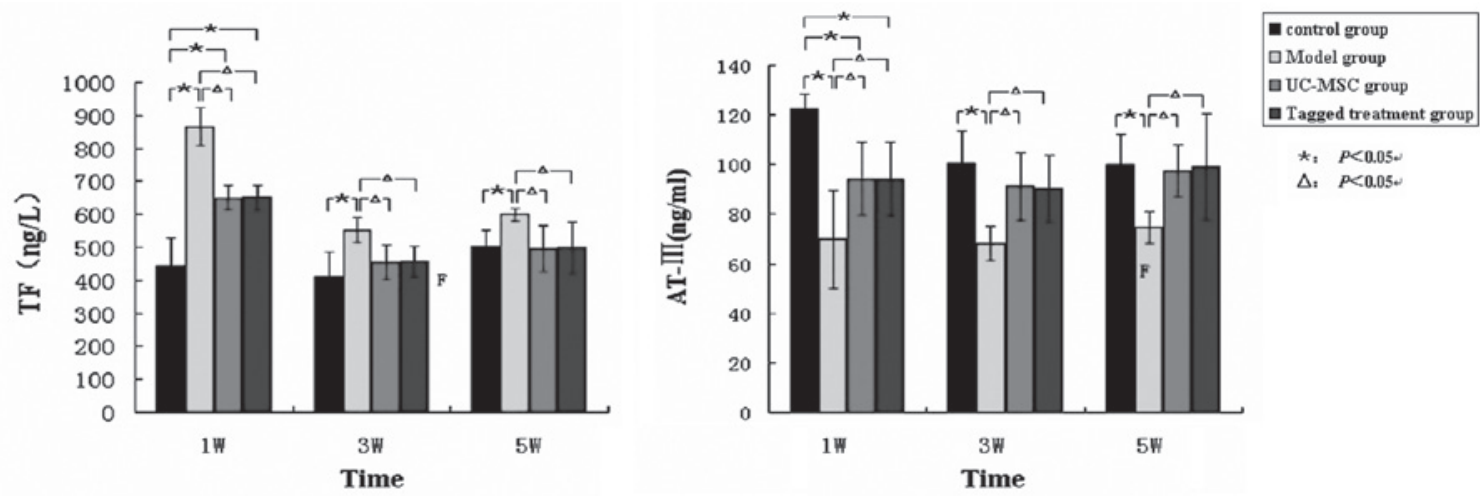

Figure 6. Comparison of the levels of TF and AT among the different groups. TF in group M was significantly higher compared with that of the hUC-MSC-treated groups and the control group, whereas the AT in the model group was significantly lower compared with that of the control and the hUC-MSC-treated group at 5 weeks post-treatment. " $\mathrm{P}<0.05$, compared with the blank control group; ${ }^{\wedge} \mathrm{P}<0.05$, compared with the hUC-MSC-treated groups. TF, tissue factor; AT, antithrombin; W week; hUC-MSCs, human umbilical cord mesenchymal stem cells; W, weeks.

and the endothelial cell protein $\mathrm{C}$ receptor (18). In addition, IL-1 also promoted fibrinolysis by increasing the production of the plasminogen activator inhibitor and decreasing the production of tissue-type plasminogen activator (19). TNF- $\alpha$ is considered to regulate a wide range of biologically active polypeptides involved in synovial inflammation reaction, and it also has a direct effect on vascular endothelial cells. Furthermore, the complete IL-17 cytokine profile includes the variants IL-17A and $\mathrm{B}$, the former making an essential contribution towards the pathogenesis of RA, whereas the latter enhances the effects of TNF- $\alpha$ on the production of cytokines and chemokines (20). VEGF activates the chemotaxis of monocytes and lymphocytes, and promotes the proliferation of fibroblasts and synovial cells in addition to releasing inflammatory mediators, thereby aggravating the severity of synovitis. VEGF also increases the permeability of veins and promotes the exudation of intravascular substances. Therefore, VEGF is considered to be the most important of the vascular permeability factors (21). Taken together, the pathogenic inflammatory cytokines stimulate arthritic processes, including the destruction of the synovium, cartilage and bone, while also damaging the vascular endothelial cells and promoting the synthesis of TF. Furthermore, these inflammatory cytokines also trigger the coagulation pathway, promote blood coagulation, and enhance the activity of the fibrinolytic system. When plasminogen is degraded and AT is depleted, the body enters into a hypercoagulable state (22). 
The present study revealed that IL-1, IL-17, TNF- $\alpha$, VEGF and TF were markedly increased, whereas AT was reduced in the untreated rat model of CIA. Notably, all inflammatory cytokines, and also TF and AT, gradually returned to almost normal levels on day 35 after UC-MSC infusion. Therefore, hUC-MSCs proved capable of reducing the adverse effects of the CIA model rat immune disorders, in addition to reducing the release of inflammatory cytokines and restoring the equilibrium of the coagulation-fibrinolysis system. The possible mechanisms, which may be involved are follows: i) hUC-MSCs regulate the immune function of the CIA model rats by upregulating the Treg cells and downregulating the expression of the Th17 cells and neutrophils, subsequently restoring the balance of the immune cells by inducing immunotolerance, promoting immune modulation and reducing the release of inflammatory cytokines. ii) hUC-MSCs may migrate to the sites of inflammation by local inflammatory signals during the acute phase of inflammation and tissue damage. hUC-MSCs are subsequently able to repair the damaged joints and immune vasculitis by making cell-cell contacts (23). iii) hUC-MSCs may also produce a variety of cytokines via the paracrine pathway to improve the local microenvironment and to promote lesion recovery (24).

Taken together, the results of the present study revealed that hUC-MSCs may migrate to the sites of inflammation in the CIA rats and effectively improve the immune-associated inflammatory and prothrombotic state.

\section{Acknowledgements}

This study was supported by grants from the National Natural Science Foundation of China (no. 81270590) and the Health Department of Jiangsu Province (no. H201048).

\section{References}

1. Lee DM and Weinblatt ME: Rheumatoid arthritis. Lancet 358: 903-911, 2001.

2. Choy E: Understanding the dynamics: Pathways involved in the pathogenesis of rheumatoid arthritis. Rheumatology (Oxford) 51 (Suppl 5): v3-v11, 2012.

3. Rampersad RR, Tarrant TK, Vallanat CT, Quintero-Matthews T, Weeks MF, Esserman DA, Clark J, Di Padova F, Patel DD, Fong AM and Liu P: Enhanced Th17-cell responses render CCR2-deficient mice more susceptible for autoimmune arthritis. PLoS One 6: e25833, 2011.

4. Aksu K, Donmez A and Keser G: Inflammation-induced thrombosis: Mechanisms, disease associations and management. Curr Pharm Des 18: 1478-1493, 2012.

5. Boilard E, Nigrovic PA, Larabee K, Watts GF, Coblyn JS, Weinblatt ME, Massarotti EM, Remold-O'Donnell E, Farndale RW, Ware J and Lee DM: Platelets amplify inflammation in arthritis via collagen-dependent microparticle production. Science 327: 580-583, 2010.

6. Snowden JA, Saccardi R, Allez M, Ardizzone S, Arnold R, Cervera R, Denton C, Hawkey C, Labopin M, Mancardi G, et al: Haematopoietic SCT in severe autoimmune diseases: Updated guidelines of the European group for blood and marrow transplantation. Bone Marrow Transplant 47: 770-790, 2012.
7. Liu R, Zhang Z, Lu Z, Borlongan C, Pan J, Chen J, Qian L, Liu Z, Zhu L, Zhang J and Xu Y: Human umbilical cord stem cells ameliorate experimental autoimmune encephalomyelitis by regulating immunoinflammation and remyelination. Stem Cells Dev 22: 1053-1062, 2013.

8. Chao KC, Chao KF, Fu YS and Liu SH: Islet-like clusters derived from mesenchymal stem cells in Wharton's Jelly of the human umbilical cord for transplantation to control type 1 diabetes. PLoS One 3: e1451, 2008

9. Van de Putte LB, Tyndall A, van den Hoogen FH and Smolen JS: Hematopoietic stem cell transplants for autoimmune disease: Role of EULAR. European League Against Rheumatism. J Rheumatol Suppl 48: 98-99, 1997.

10. Xie J, Liu G, Eden HS, Ai H and Chen X: Surface-engineered magnetic nanoparticle platforms for cancer imaging and therapy. Acc Chem Res 44: 883-892, 2011.

11. Lin CM, Gu J, Zhang Y, Shen LJ, Ma L, Ni J, Wang ZQ and Wu W: Effect of UC-MSCs on inflammation and thrombosis of the rats with collagen type II induced arthritis. Zhonghua Xue Ye Xue Za Zhi 33: 215-219, 2012 (In Chinese).

12. Lange F, Bajtner E, Rintisch C, Nandakumar KS, Sack U and Holmdahl R: Methotrexate ameliorates T cell dependent autoimmune arthritis and encephalomyelitis but not antibody induced or fibroblast induced arthritis. Ann Rheum Dis 64: 599-605, 2005.

13. Mahmoudi M, Sant S, Wang B, Laurent S and Sen T: Superparamagnetic iron oxide nanoparticles (SPIONs): Development, surface modification and applications in chemotherapy. Adv Drug Deliv Rev 63: 24-46, 2011.

14. Lefevre S, Ruimy D, Jehl F, Neuville A, Robert P, Sordet C, Ehlinger M, Dietemann JL and Bierry G: Septic arthritis: Monitoring with USPIO enhanced macrophage MR imaging. Radiology 258: 722-728, 2011.

15. Hu SL, Zhang JQ, Hu X, Hu R, Luo HS, Li F, Xia YZ, Li JT, Lin JK, Zhu G and Feng H: In vitro labeling of human umbilical cord mesenchymal stem cells with superparamagnetic iron oxide nanoparticles. J Cell Biochem 108: 529-535, 2009.

16. Janic B, Rad AM, Jordan EK, Iskander AS, Ali MM, Varma NR, Frank JA and Arbab AS: Optimization and validation of FePro cell labeling method. PLoS One 4: e5873, 2009.

17. De Almeida DE, Ling S, Pi X, Hartmann-Scruggs AM, Pumpens P and Holoshitz J: Immune dysregulation by the rheumatoid arthritis shared epitope. J Immunol 185: 1927-1934, 2010.

18. Liu X, Ye F, Xiong H, Hu DN, et al: IL-1 $\beta$ induces IL-6 production in retinal Müller cells predominantly through the activation of p38 MAPK/NF- $\mathrm{B}$ signaling pathway. Exp Cell Res 331: 223-231, 2015.

19. Joseph L, Fink LM and Hauer-Jensen M: Cytokines in coagulation and thrombosis: A preclinical and clinical review. Blood Coagul Fibrinolysis 13: 105-116, 2002.

20. Kouri VP, Olkkonen J, Ainola M, Li TF, Björkman L, Konttinen YT and Mandelin J: Neutrophils produce interleukin-17B in rheumatoid synovial tissue. Rheumatology (Oxford) 53: 39-47, 2014.

21. Noma H, Mimura T and Eguchi S: Association of inflammatory factors with macular edema in branch retinal vein occlusion. JAMA Ophthalmol 131: 160-165, 2013.

22. Undas A, Gissel M, Kwasny-Krochin B, Gluszko P, Mann KG and Brummel-Ziedins KE: Thrombin generation in rheumatoid arthritis: Dependence on plasma factor composition. Thromb Haemost 104: 224-230, 2010.

23. Sun L, Akiyama K, Zhang H, Hou Y, Zhao S, Xu T, Le A and Shi S: Mesenchymal stem cell transplantation reverses multiorgan dysfunction in systemic lupus erythematosus mice and humans. Stem Cells 27: 1421-1432, 2009.

24. Gu ZF, Akiyama K, Ma X, Zhang H, Feng X, Yao G, Hou Y, Lu L, Gilkeson GS, Silver RM, et al: Transplantation of umbilical cord mesenchymal stem cells alleviates lupus nephritis in MRL/lpr mice. Lupus 19: 1502-1514, 2010. 